\title{
On partial regularity for the steady Hall magnetohydrodynamics system
}

\author{
Dongho Chae* and Jörg Wolf ${ }^{\dagger}$ \\ *Department of Mathematics \\ Chung-Ang University \\ Seoul 156-756, Republic of Korea \\ e-mail: dchae@cau.ac.kr \\ and \\ $\dagger$ Department of Financial Engineering \\ Ajou University \\ Suwon 443-749, Republic of Korea \\ e-mail: jwolf@math.hu-berlin.de
}

\begin{abstract}
We study partial regularity of suitable weak solutions of the steady Hall magnetohydrodynamics equations in a domain $\Omega \subset \mathbb{R}^{3}$. In particular we prove that the set of possible singularities of the suitable weak solution has Hausdorff dimension at most one. Moreover, in the case $\Omega=\mathbb{R}^{3}$, we show that the set of possible singularities is compact.
\end{abstract}

AMS Subject Classification Number: 35Q35, 35Q85,76W05

keywords: steady Hall-MHD equations, parial regularity

\section{Introduction}

The resistive incompressible Hall magnetohydrodynamics(Hall-MHD) is described by he following equations:

$$
\left\{\begin{array}{l}
\frac{\partial \boldsymbol{u}}{\partial t}+\boldsymbol{u} \cdot \nabla \boldsymbol{u}+\nabla p=(\nabla \times \boldsymbol{B}) \times \boldsymbol{B}+\nu \Delta \boldsymbol{u}+\boldsymbol{f} \\
\frac{\partial \boldsymbol{B}}{\partial t}-\nabla \times(\boldsymbol{u} \times \boldsymbol{B})+\nabla \times((\nabla \times \boldsymbol{B}) \times \boldsymbol{B})=\mu \Delta B+\nabla \times \boldsymbol{g} \\
\nabla \cdot \boldsymbol{u}=0, \nabla \cdot \boldsymbol{B}=0
\end{array}\right.
$$

where 3D vector fields $\boldsymbol{u}=\boldsymbol{u}(x, t), \boldsymbol{B}=\boldsymbol{B}(x, t)$ are the fluid velocity and the magnetic field respectively. The scalar field $p=p(x, t)$ is the pressure, while the positive 
constants $\nu$ and $\mu$ represent the viscosity and the magnetic resistivity respectively. The given vector fields $\boldsymbol{f}$ and $\nabla \times \boldsymbol{g}$ are external forces on the magnetically charged fluid flows. Historically, the Hall-MHD system was first considered by Lighthill([15]). Compared with the usual MHD system, the Hall-MHD system contains the extra term $\nabla \times((\nabla \times \boldsymbol{B}) \times \boldsymbol{B})$, called the Hall term. The inclusion of this term is essential in understanding the problem of magnetic reconnection, which corresponds to the change of the topology of magnetic field lines. This phenomena of magnetic reconnection is really observed, for example, in space plasma([10, 12]), star formation([22]) and neutron $\operatorname{star}([19])$. For the other physical features related to the Hall-MHD we refer [20, 21], while for a comprehensive review of the physical aspect of the equations we refer [17. Since the Hall term involves the second order derivative of the magnetic field, it becomes important when the magnetic shear is very large, and this occurs during the reconnection procedure. In the laminar flows this term is small compared with the other term, and can be neglected, which is the case of the usual MHD.

Since the Hall term is quadratically nonlinear, containing the second order derivative, it causes major difficulties in the mathematical study of the Hall-MHD system, and only recently the rigorous results on the Cauchy problem appeared. In [1] the authors proved the global existence of weak solutions, while the local in time wellposedness as well as the global in time well-posedness for small initial data was proved in [4]. This later result was refined in [5]. In the case of $\mu=0$ it is proved in [7] that the Cauchy problem is not globally in time well-posed, rigorously verifying the numerical experiment of [8]. For a special axially symmetric initial data the authors of [9] proved the global well-posedness of the system. In [6] the long time behaviors of the solution were also studied. Since the Hall-MHD system has more complicated structure than the usual MHD system and the Navier-Stokes equations, the study of full regularity of weak solutions would be extremely difficult. Therefore, it might be reasonable to begin with the partial regularity, similarly to the case of the Navier-Stokes equations, the partial regularity of which was studied e.g. in [18, 22, 14, 16, 23]. In the time-dependent problem, mainly due to the difficulty of defining the correct localized energy inequality for a suitable weak solutions we concentrate the partial regularity problem of the steady Hall-MHD system. Contrary to the case of the Navier-Stokes equations and the usual MHD system the full regularity of the steady weak solutions is difficult to deduce. Instead, we prove that the set of possible singularity of the steady suitable weak solution of the Hall-MHD system has Hausdorff dimension at most 1(see Remark 5.2 and Theorem 5.3 below). Moreover, for a steady suitable weak solution on $\mathbb{R}^{3}$ the set of possible singularity is a compact set(see Corollary 7.3 below). The partial regularity of the time dependent problem will be studied elsewhere. 


\section{Weak solution and higher regularity of $u$}

We consider the following steady Hall-MHD system in $\mathbb{R}^{3}$.

$$
\begin{aligned}
(\boldsymbol{u} \cdot \nabla) \boldsymbol{u}-\Delta \boldsymbol{u} & =-\nabla p+(\nabla \times \boldsymbol{B}) \times \boldsymbol{B}+\boldsymbol{f} \\
\nabla \times(\boldsymbol{B} \times \boldsymbol{u})-\Delta \boldsymbol{B} & =-\nabla \times((\nabla \times \boldsymbol{B}) \times \boldsymbol{B})+\nabla \times \boldsymbol{g}, \\
\nabla \cdot \boldsymbol{u} & =0, \nabla \cdot \boldsymbol{B}=0 .
\end{aligned}
$$

We note that we set $\mu=\nu=1$ for convenience. As $(\boldsymbol{u} \cdot \nabla) \boldsymbol{u}=(\nabla \times \boldsymbol{u}) \times \boldsymbol{u}+\frac{1}{2}|\boldsymbol{u}|^{2}$, (2.1) turns into

$$
(\nabla \times \boldsymbol{u}) \times \boldsymbol{u}-\Delta \boldsymbol{u}=-\nabla\left(p+\frac{|\boldsymbol{u}|^{2}}{2}\right)+(\nabla \times \boldsymbol{B}) \times \boldsymbol{B}+\boldsymbol{f} \quad \text { in } \quad \mathbb{R}^{3} .
$$

Applying $\nabla \times$ to the both sides of the above, we get

$$
\nabla \times(\boldsymbol{\omega} \times \boldsymbol{u})-\Delta \boldsymbol{\omega}=\nabla \times((\nabla \times \boldsymbol{B}) \times \boldsymbol{B})+\nabla \times \boldsymbol{f} \text { in } \mathbb{R}^{3},
$$

where $\boldsymbol{\omega}$ stands for the vorticity $\nabla \times \boldsymbol{u}$. Taking the sum of $(2.2)$ and (2.5), we are led to

$$
\nabla \times(\boldsymbol{V} \times \boldsymbol{u})-\Delta \boldsymbol{V}=\nabla \times(\boldsymbol{f}+\boldsymbol{g}) \text { in } \mathbb{R}^{3}
$$

where

$$
\boldsymbol{V}=\boldsymbol{B}+\boldsymbol{\omega}
$$

As $\nabla \cdot \boldsymbol{V}=0$, there exists a solenoidal potential $\boldsymbol{v}$ such that $\nabla \times \boldsymbol{v}=\boldsymbol{V}$. From (2.6) we deduce that $\boldsymbol{v}$ solves the system in $\mathbb{R}^{3}$,

$$
\begin{aligned}
\nabla \cdot \boldsymbol{v} & =0 \\
(\boldsymbol{v} \cdot \nabla) \boldsymbol{v}-\Delta \boldsymbol{v} & =-\nabla \pi+(\nabla \times \boldsymbol{v}) \times \boldsymbol{b}+\boldsymbol{f}+\boldsymbol{g},
\end{aligned}
$$

where $\boldsymbol{b}=\boldsymbol{v}-\boldsymbol{u}$. Clearly, $\nabla \times \boldsymbol{b}=\boldsymbol{B}$.

Definition 2.1. Let $\boldsymbol{f} \in L^{6 / 5}$ and $\boldsymbol{g} \in L^{2}$. We say $(\boldsymbol{u}, p, \boldsymbol{B}) \in \dot{W}^{1,2} \times L_{\text {loc }}^{2} \times \dot{W}^{1,2}$ is a weak solution to (2.3)-(2.2) if

$$
\begin{aligned}
& \int_{\mathbb{R}^{3}} \nabla \boldsymbol{u}: \nabla \boldsymbol{\varphi}-\boldsymbol{u} \otimes \boldsymbol{u}: \nabla \boldsymbol{\varphi} d x \\
& \quad=\int_{\mathbb{R}^{3}} p \nabla \cdot \boldsymbol{\varphi} d x+\int_{\mathbb{R}^{3}}((\nabla \times \boldsymbol{B}) \times \boldsymbol{B}) \cdot \boldsymbol{\varphi} d x+\int_{\mathbb{R}^{3}} \boldsymbol{f} \cdot \boldsymbol{\varphi} d x \\
& \int_{\mathbb{R}^{3}} \nabla \boldsymbol{B}: \nabla \boldsymbol{\varphi}+\boldsymbol{B} \times \boldsymbol{u} \cdot \nabla \times \boldsymbol{\varphi} d x
\end{aligned}
$$

$$
=-\int_{\mathbb{R}^{3}}((\nabla \times \boldsymbol{B}) \times \boldsymbol{B}) \cdot \nabla \times \boldsymbol{\varphi} d x+\int_{\mathbb{R}^{3}} \boldsymbol{g} \cdot \nabla \times \boldsymbol{\varphi} d x
$$

for all $\varphi \in C_{\mathrm{c}}^{\infty}$. Here $\dot{W}^{1,2}$ stands for the homogeneous Sobolev space. 
Remark 2.2. Since $\boldsymbol{B} \in \dot{W}^{1,2} \hookrightarrow L^{6}$, by the Calderón-Zygmund inequality and Sobolev's embedding theorem we infer $\boldsymbol{b} \in L_{\text {loc }}^{\infty}$. In particular, having $\boldsymbol{V} \in L_{\text {loc }}^{2}$ we easily deduce that $\boldsymbol{v} \in W_{\text {loc }}^{1,2}$ and $\pi \in L_{\text {loc }}^{2}$. Furthermore, $(\boldsymbol{v}, \pi)$ satisfies the following integral identity for all $\varphi \in C_{\mathrm{c}}^{\infty}$

$$
\begin{aligned}
& \int_{\mathbb{R}^{3}} \nabla \boldsymbol{v}: \nabla \boldsymbol{\varphi}-\boldsymbol{v} \otimes \boldsymbol{v}: \nabla \boldsymbol{\varphi} d x \\
& \quad=\int_{\mathbb{R}^{3}} \pi \nabla \cdot \boldsymbol{\varphi} d x+\int_{\mathbb{R}^{3}}((\nabla \times \boldsymbol{v}) \times \boldsymbol{b}) \cdot \boldsymbol{\varphi} d x+\int_{\mathbb{R}^{3}}(\boldsymbol{f}+\boldsymbol{g}) \cdot \boldsymbol{\varphi} d x .
\end{aligned}
$$

By using standard regularity methods we get the following

Theorem 2.3. Let $\boldsymbol{f} \in L^{6 / 5}$ and $\boldsymbol{g} \in L^{2}$. Let $(\boldsymbol{u}, p, \boldsymbol{B}) \in \dot{W}^{1,2} \times L_{\mathrm{loc}}^{2} \times \dot{W}^{1,2}$ be a weak solution to (2.3)-(2.2). Suppose, $\boldsymbol{f}, \boldsymbol{g} \in L_{\mathrm{loc}}^{q}(\Omega)$ for some $\frac{6}{5}<q<+\infty$ and for an open set $\Omega \subset \mathbb{R}^{3}$. Then

$$
\boldsymbol{V} \in W_{\mathrm{loc}}^{1, q}(\Omega), \quad \boldsymbol{v} \in W_{\mathrm{loc}}^{2, q}(\Omega), \quad \boldsymbol{u} \in W_{\mathrm{loc}}^{2, q \wedge 2}(\Omega),
$$

where $q \wedge 2=\min \{q, 2\}$.

Proof First, assume $\frac{3}{2} \leq q<+\infty$. Since $\boldsymbol{u} \in L^{6}$ and $\boldsymbol{V} \in L_{\text {loc }}^{2}$, we see that $-\boldsymbol{V} \times \boldsymbol{u}+$ $\boldsymbol{f}+\boldsymbol{g} \in L_{\mathrm{loc}}^{3 / 2}(\Omega)$. Observing (2.6), by the aid of Calderón-Zygmund's inequality and Sobolev's embedding theorem we find $\boldsymbol{V} \in W_{\mathrm{loc}}^{1,3 / 2}(\Omega) \subset L_{\mathrm{loc}}^{3}(\Omega)$. As $\boldsymbol{\omega}=\boldsymbol{V}-\boldsymbol{B}$, this implies $\boldsymbol{\omega} \in L_{\text {loc }}^{3}(\Omega)$. Taking into account $\nabla \cdot \boldsymbol{u}=0$, we get $\boldsymbol{u} \in W_{\text {loc }}^{1,3}(\Omega)$. Once more appealing to Sobolev's embedding therorem, we obtain $\boldsymbol{u} \in L_{\text {loc }}^{s}(\Omega)$ for all $1 \leq s<+\infty$, and thus $-\boldsymbol{V} \times \boldsymbol{u}+\boldsymbol{f}+\boldsymbol{g} \in L_{\mathrm{loc}}^{q}(\Omega)$. Again applying Calderón-Zygmund's inequality, we see that $\boldsymbol{V} \in W_{\text {loc }}^{1, q}(\Omega)$. As $\nabla \cdot \boldsymbol{v}=0$ from the last statement we infer $\boldsymbol{v} \in W_{\text {loc }}^{2, q}(\Omega)$. Finally, recalling $\boldsymbol{\omega}=\boldsymbol{V}-\boldsymbol{B}$ and $\boldsymbol{B} \in W_{\mathrm{loc}}^{1,2}(\Omega)$, we obtain $\boldsymbol{\omega} \in W_{\mathrm{loc}}^{1, q \wedge 2}(\Omega)$.

In case $\frac{6}{5}<q<\frac{3}{2}$, we immediately get $-\boldsymbol{V} \times \boldsymbol{u}+\boldsymbol{f}+\boldsymbol{g} \in L_{\mathrm{loc}}^{q}(\Omega)$, and the assertion can be proved as in the previous case.

\section{Caccioppoli-type inequality for $B$}

Suppose $\boldsymbol{f}, \boldsymbol{g} \in L_{\text {loc }}^{2}(\Omega)$ for some open set $\Omega \subset \mathbb{R}^{3}$. As it has been proved in Section 2 we get $\boldsymbol{u} \in W_{\text {loc }}^{2,2}(\Omega)$ if $(\boldsymbol{u}, p, \boldsymbol{B})$ is a weak solution to the steady Hall-MHD system. By means of Sobolev's embedding theorem this implies $\boldsymbol{u} \in L_{\text {loc }}^{\infty}(\Omega)$. Accordingly, $-\boldsymbol{B} \times \boldsymbol{u}+\boldsymbol{g} \in L_{\mathrm{loc}}^{2}(\Omega)$.

Thus, for the sake of generality in the present and next section we study the local regularity for the following general model. Let $\Omega \subset \mathbb{R}^{3}$ be a domain. We consider the system

$$
-\Delta \boldsymbol{B}=-\nabla \times((\nabla \times \boldsymbol{B}) \times \boldsymbol{B})+\nabla \times \boldsymbol{F} \text { in } \Omega .
$$

We start our discussion with the following notion of a weak solution to (3.1). 
Definition 3.1. Let $\boldsymbol{F} \in L_{\text {loc }}^{2}(\Omega)$. (i) A vector function $\boldsymbol{B} \in W_{\text {loc }}^{1,2}(\Omega)$ is said to be a weak solution to (3.1) if

$$
\int_{\Omega} \nabla \boldsymbol{B}: \nabla \boldsymbol{\varphi} d x=-\int_{\Omega}(\nabla \times \boldsymbol{B}) \times \boldsymbol{B} \cdot \nabla \times \boldsymbol{\varphi} d x+\int_{\Omega} \boldsymbol{F} \cdot \nabla \times \boldsymbol{\varphi} d x
$$

for all $\varphi \in C_{\mathrm{c}}^{\infty}(\Omega)$.

(ii) A weak solution $\boldsymbol{B}$ to (3.1) is called a suitable weak solution to (3.1) if, in addition, the following local energy inequality holds:

$$
\begin{aligned}
& \int_{\Omega} \phi|\nabla \boldsymbol{B}|^{2} d x \\
& \leq \frac{1}{2} \int_{\Omega} \Delta \phi|\boldsymbol{B}|^{2} d x-\int_{\Omega}(\nabla \times \boldsymbol{B}) \times \boldsymbol{B} \cdot(\boldsymbol{B} \times \nabla \phi) d x \\
& \quad+\int_{\Omega}(\phi \boldsymbol{F} \cdot \nabla \times \boldsymbol{B}+\boldsymbol{F} \cdot \boldsymbol{B} \times \nabla \phi) d x
\end{aligned}
$$

for all non-negative $\phi \in C_{\mathrm{c}}^{\infty}(\Omega)$.

Remark 3.2. Let $\boldsymbol{B}$ be a suitable weak solution to (3.1). Then for every constant vector $\Lambda \in \mathbb{R}^{3}$ there holds

$$
\begin{aligned}
& \int_{\Omega} \phi|\nabla \boldsymbol{B}|^{2} d x \\
& \leq \frac{1}{2} \int_{\Omega} \Delta \phi|\boldsymbol{B}-\boldsymbol{\Lambda}|^{2} d x-\int_{\Omega}(\nabla \times \boldsymbol{B}) \times \boldsymbol{B} \cdot((\boldsymbol{B}-\boldsymbol{\Lambda}) \times \nabla \phi) d x \\
& \quad+\int_{\Omega}\{\phi \boldsymbol{F} \cdot \nabla \times \boldsymbol{B}+\boldsymbol{F} \cdot(\boldsymbol{B}-\boldsymbol{\Lambda}) \times \nabla \phi\} d x
\end{aligned}
$$

for all non-negative $\phi \in C_{\mathrm{c}}^{\infty}(\Omega)$. This can be readily seen by combining (3.3) and (3.2) with $\varphi=\phi \boldsymbol{\Lambda}$.

Now, we state the following Caccioppoli-type inequality

Lemma 3.3. Let $\boldsymbol{F} \in L_{\mathrm{loc}}^{2}(\Omega)$. Let $\boldsymbol{B} \in W_{\mathrm{loc}}^{1,2}(\Omega)$ be a suitable weak solution to (3.1). Then for every ball $B_{r}=B_{r}\left(x_{0}\right) \subset \subset \Omega$ and $0<\rho<r$ there holds

$$
\begin{aligned}
& \frac{1}{r} \int_{B_{\rho}}|\nabla \boldsymbol{B}|^{2} d x \\
& \quad \leq \frac{c r^{2}}{(r-\rho)^{2}}\left(1+\left|\boldsymbol{B}_{r, x_{0}}\right|^{2}\right) f_{B_{r}}\left|\boldsymbol{B}-\boldsymbol{B}_{r, x_{0}}\right|^{2} d x+\frac{c r^{2}}{(r-\rho)^{2}} f_{B_{r}}\left|\boldsymbol{B}-\boldsymbol{B}_{r, x_{0}}\right|^{4} d x \\
& \quad+\frac{c}{r}\|\boldsymbol{F}\|_{2, B_{r}}^{2},
\end{aligned}
$$


where $\boldsymbol{B}_{r, x_{0}}$ stands for the mean value

$$
f_{B_{r}} \boldsymbol{B} d x:=\frac{1}{\operatorname{meas}\left(B_{r}\right)} \int_{B_{r}\left(x_{0}\right)} \boldsymbol{B} d x
$$

and $c=$ const $>0$ denotes a universal constant.

Proof Let $B_{r}=B_{r}\left(x_{0}\right) \subset \subset \Omega$ be a fixed ball. Given $\rho \in(0, r)$, we consider a cut-off function $\zeta$ defined as $\zeta \in C_{\mathrm{c}}^{\infty}\left(B_{r}\right)$ such that $0 \leq \zeta \leq 1$ in $\mathbb{R}^{3}, \zeta \equiv 1$ on $B_{\rho}$ and $|\nabla \zeta|^{2}+\left|\nabla^{2} \zeta\right| \leq c(r-\rho)^{-2}$ in $\mathbb{R}^{3}$. From (3.4) with $\phi=\zeta^{2}$ and $\boldsymbol{\Lambda}=\boldsymbol{B}_{r, x_{0}}$ we obtain the following Caccioppoli-type inequality

$$
\begin{aligned}
& \int_{B_{r}} \zeta^{2}|\nabla \boldsymbol{B}|^{2} d x \\
& \leq \frac{c}{(r-\rho)^{2}} \int_{B_{r}}\left|\boldsymbol{B}-\boldsymbol{B}_{r, x_{0}}\right|^{2} d x+c \int_{B_{r}}|\boldsymbol{F}|^{2} d x \\
& \quad+\frac{c}{r-\rho} \int_{B_{r}} \zeta|\nabla \boldsymbol{B}||\boldsymbol{B}|\left|\boldsymbol{B}-\boldsymbol{B}_{r, x_{0}}\right| d x \\
& =\frac{c}{(r-\rho)^{2}} \int_{B_{r}}\left|\boldsymbol{B}-\boldsymbol{B}_{r, x_{0}}\right|^{2} d x+c \int_{B_{r}}|\boldsymbol{F}|^{2} d x+J .
\end{aligned}
$$

Applying Hölder's and Young's inequality, we estimate

$$
\begin{aligned}
J & \leq \frac{c}{(r-\rho)^{2}} \int_{B_{r}}|\boldsymbol{B}|^{2}\left|\boldsymbol{B}-\boldsymbol{B}_{r, x_{0}}\right|^{2} d x+\frac{1}{2} \int_{B_{r}} \zeta^{2}|\nabla \boldsymbol{B}|^{2} d x \\
& \leq \frac{c}{(r-\rho)^{2}}\left|\boldsymbol{B}_{r, x_{0}}\right|^{2} \int_{B_{r}}\left|\boldsymbol{B}-\boldsymbol{B}_{r, x_{0}}\right|^{2} d x+\frac{c}{(r-\rho)^{2}} \int_{B_{r}}\left|\boldsymbol{B}-\boldsymbol{B}_{r, x_{0}}\right|^{4} d x \\
& +\frac{1}{2} \int_{B_{r}} \zeta^{2}|\nabla \boldsymbol{B}|^{2} d x .
\end{aligned}
$$

Inserting the above estimate of $J$ into the right-hand side of (3.6), and dividing the resulting estimate by $r$, we are led to

$$
\begin{aligned}
& \frac{1}{r} \int_{B_{\rho}}|\nabla \boldsymbol{B}|^{2} d x \\
& \quad \leq \frac{c r^{2}}{(r-\rho)^{2}}\left(1+\left|\boldsymbol{B}_{r, x_{0}}\right|^{2}\right) f_{B_{r}}\left|\boldsymbol{B}-\boldsymbol{B}_{r, x_{0}}\right|^{2} d x+\frac{c r^{2}}{(r-\rho)^{2}} f_{B_{r}}\left|\boldsymbol{B}-\boldsymbol{B}_{r, x_{0}}\right|^{4} d x \\
& \quad+\frac{c}{r}\|\boldsymbol{F}\|_{2, B_{r}}^{2} .
\end{aligned}
$$

Whence, the claim. 
Remark 3.4. Setting

$$
E(r)=E\left(r, x_{0}\right)=\left(f_{B_{r}\left(x_{0}\right)}\left|\boldsymbol{B}-\boldsymbol{B}_{r ; x_{0}}\right|^{4} d x\right)^{1 / 4} \quad 0<r<\operatorname{dist}\left(x_{0}, \partial \Omega\right),
$$

(3.5) becomes

$$
\left(\frac{1}{r} \int_{B_{\rho}}|\nabla \boldsymbol{B}|^{2} d x\right)^{1 / 2} \leq \frac{c r}{r-\rho}\left\{\left(1+\left|\boldsymbol{B}_{r, x_{0}}\right|\right) E(r)+E(r)^{2}\right\}+\frac{c}{r^{1 / 2}}\|\boldsymbol{F}\|_{2, B_{r}} .
$$

\section{Blow-up}

We begin our discussion with the following fundamental estimate for solutions to the model problem, which will be used in the blow-up lemma below.

Lemma 4.1. Let $\boldsymbol{\Lambda} \in \mathbb{R}^{3}$. Let $\boldsymbol{W} \in L^{4}\left(B_{1}\right) \cap W_{\text {loc }}^{1,2}\left(B_{1}\right)$ be a weak solution to

$$
-\Delta \boldsymbol{W}=-\nabla \times((\nabla \times \boldsymbol{W}) \times \boldsymbol{\Lambda}) \text { in } B_{1},
$$

i.e.

$$
\int_{B_{1}} \nabla \boldsymbol{W}: \nabla \boldsymbol{\Phi} d x=-\int_{B_{1}}((\nabla \times \boldsymbol{W}) \times \boldsymbol{\Lambda}) \cdot \nabla \times \boldsymbol{\Phi} d x
$$

for all $\boldsymbol{\Phi} \in W^{1,2}\left(B_{1}\right)$ with $\operatorname{supp}(\boldsymbol{\Phi}) \subset \subset B_{1}$. Then,

$$
\left(f_{B_{\tau}}\left|\boldsymbol{W}-\boldsymbol{W}_{B_{\tau}}\right|^{4} d x\right)^{1 / 4} \leq C_{0} \tau\left(1+|\boldsymbol{\Lambda}|^{3}\right)\left(f_{B_{1}}\left|\boldsymbol{W}-\boldsymbol{W}_{B_{1}}\right|^{4} d x\right)^{1 / 4} \quad \forall 0<\tau<1,
$$

where $C_{0}>0$ denotes a universal constant.

Proof Since the assertion is trivial for $\frac{1}{2}<\tau<1$, we may assume that $0<\tau<\frac{1}{2}$. Let $\zeta \in C_{\mathrm{c}}^{\infty}\left(B_{1}\right)$ be a suitable cut-off function for $B_{1 / 2}$, i. e. $0 \leq \zeta \leq 1$ in $B_{1}$ and $\zeta \equiv 1$ on $B_{1 / 2}$. In (4.2) inserting the admissible test function $\boldsymbol{\Phi}=\boldsymbol{\zeta}^{2 m}\left(\boldsymbol{W}-\boldsymbol{W}_{B_{1}}\right)(m \in \mathbb{N})$, by using Cauchy-Schwarz's inequality along with Young's inequality we obtain

$$
\int_{B_{1}} \zeta^{2 m}|\nabla \boldsymbol{W}|^{2} d x \leq c\left(1+|\boldsymbol{\Lambda}|^{2}\right) \int_{B_{1}} \zeta^{2 m-2}\left|\boldsymbol{W}-\boldsymbol{W}_{B_{1}}\right|^{2} d x
$$

If $\boldsymbol{W}$ is smooth in $B_{1}$, since (4.1) is a linear system, the same inequality holds for $D^{\alpha} \boldsymbol{W}$ in place of $\boldsymbol{W}$ for any multi-index $\alpha$. By a standard mollifying argument together with Sobolev's embedding theorem we see that $\boldsymbol{W}$ is smooth. In particular, in (4.4) putting $m=3$ it follows that

$$
\int_{B_{1}} \zeta^{6}\left|D^{\alpha} \boldsymbol{W}\right|^{2} d x \leq c\left(1+|\boldsymbol{\Lambda}|^{6}\right) \int_{B_{1}}\left|\boldsymbol{W}-\boldsymbol{W}_{B_{1}}\right|^{2} d x \quad \forall|\alpha| \leq 3 .
$$


By means of Sobolev's embedding theorem and Jensen's inequality we get

$$
\|\nabla \boldsymbol{W}\|_{\infty, B_{1 / 2}}^{4} \leq c\left(1+|\boldsymbol{\Lambda}|^{12}\right) \int_{B_{1}}\left|\boldsymbol{W}-\boldsymbol{W}_{B_{1}}\right|^{4} d x
$$

Applying Poincaré's inequality, we obtain

$$
f_{B_{\tau}}\left|\boldsymbol{W}-\boldsymbol{W}_{B_{\tau}}\right|^{4} d x \leq c \tau^{4}\|\nabla \boldsymbol{W}\|_{\infty, B_{1 / 2}}^{4}
$$

Combination of (4.6) and (4.7) yields the desired estimate.

In our discussion below we make use of the notion of the Morrey space. We say $\boldsymbol{F} \in \mathcal{M}_{\mathrm{loc}}^{p, \lambda}(\Omega)$ if for all $K \subset \subset \Omega$

$$
[\boldsymbol{F}]_{\mathcal{M}^{p, \lambda}, K}^{p}:=\sup \left\{r^{-\lambda} \int_{B_{r}\left(x_{0}\right)}|\boldsymbol{F}|^{p} d x \mid x_{0} \in K, 0<r \leq \operatorname{dist}(K, \partial \Omega)\right\}<+\infty .
$$

Lemma 4.2. Let $\boldsymbol{F} \in \mathcal{M}_{\mathrm{loc}}^{2, \lambda}(\Omega)$ for some $1<\lambda \leq 3$. For every $0<\tau<\frac{1}{2}, 0<$ $M<+\infty, K \subset \subset \Omega$ and $0<\alpha<\frac{\lambda-1}{2}$, there exist positive numbers $\varepsilon_{0}=\varepsilon_{0}(\tau, M, K, \alpha)$ and $R_{0}=R_{0}(\tau, M, K, \alpha)<\operatorname{dist}(K, \partial \Omega)$ such that, if $\boldsymbol{B} \in W_{\text {loc }}^{1,2}(\Omega)$ is a suitable weak solution to (3.1), and for $x_{0} \in K$ and $0<R \leq R_{0}$ the condition

$$
\left|\boldsymbol{B}_{R, x_{0}}\right| \leq M, \quad E\left(R, x_{0}\right)+R^{\alpha} \leq \varepsilon_{0}
$$

is fulfilled, then

$$
E\left(\tau R, x_{0}\right) \leq 2 \tau C_{0}\left(1+M^{3}\right)\left(E\left(R, x_{0}\right)+R^{\alpha}\right)
$$

where $C_{0}>0$ stands for the constant which appears on the right-hand side of (4.3).

Proof Assume the assertion of the Lemma is not true. Then there exist $0<\tau<\frac{1}{2}, 0<$ $M<+\infty, K \subset \subset \Omega$ and $0<\alpha<\frac{\lambda-1}{2}$ together with a sequence $\boldsymbol{B}^{(k)} \in W_{\text {loc }}^{1,2}(\Omega)$ being suitable weak solutions to (3.1) as well as sequences $x_{k} \in K, 0<R_{k}<\operatorname{dist}(K, \partial \Omega)$ and $\varepsilon_{k} \rightarrow 0$ as $k \rightarrow+\infty$ such that

$$
\left|\boldsymbol{B}_{R_{k}, x_{k}}^{(k)}\right| \leq M, \quad E_{k}\left(R_{k}, x_{k}\right)+R_{k}^{\alpha}=\varepsilon_{k}
$$

and

$$
E_{k}\left(\tau R_{k}, x_{k}\right)>2 \tau C_{0}\left(1+M^{3}\right)\left(E_{k}\left(R_{k}, x_{k}\right)+R_{k}^{\alpha}\right)
$$

Here we have used the notation

$$
E_{k}\left(r, x_{0}\right)=\left(f_{B_{r}\left(x_{0}\right)}\left|\boldsymbol{B}^{(k)}-\boldsymbol{B}_{r, x_{0}}^{(k)}\right|^{4} d x\right)^{1 / 4}, \quad x_{0} \in K, 0<r \leq \operatorname{dist}(K, \partial \Omega) .
$$

Note that (4.10) yields $R_{k} \rightarrow 0$ as $k \rightarrow+\infty$. 
Next, define

$$
\begin{aligned}
\boldsymbol{W}_{k}(y) & =\frac{1}{\varepsilon_{k}}\left(\boldsymbol{B}^{(k)}\left(x_{k}+R_{k} y\right)-\boldsymbol{B}_{R_{k}, x_{k}}^{(k)}\right), \\
\boldsymbol{F}_{k}(y) & =\boldsymbol{F}\left(x_{k}+R_{k} y\right), \quad y \in B_{1}(0)
\end{aligned}
$$

$(k \in \mathbb{N})$. Furthermore, set

$$
\mathscr{E}_{k}(\sigma)=\left(f_{B_{\sigma}}\left|\boldsymbol{W}_{k}-\left(\boldsymbol{W}_{k}\right)_{B_{\sigma}}\right|^{4} d y\right)^{1 / 4}, \quad 0<\sigma \leq 1 .
$$

Then (4.10) and (4.11) turn into

$$
\left|\boldsymbol{B}_{R_{k}, x_{k}}^{(k)}\right| \leq M, \quad \mathscr{E}_{k}(1)+\frac{R_{k}^{\alpha}}{\varepsilon_{k}}=1
$$

and

$$
\mathscr{E}_{k}(\tau)>2 \tau C_{0}\left(1+M^{3}\right)\left(\mathscr{E}_{k}(1)+\frac{R_{k}^{\alpha}}{\varepsilon_{k}}\right)=2 \tau C_{0}\left(1+M^{3}\right)
$$

respectively.

Using the chain rule, we find that (3.1) transforms into

$$
\begin{gathered}
-\Delta \boldsymbol{W}_{k}=-\varepsilon_{k} \nabla \times\left(\left(\nabla \times \boldsymbol{W}_{k}\right) \times \boldsymbol{W}_{k}\right)-\nabla \times\left(\left(\nabla \times \boldsymbol{W}_{k}\right) \times \boldsymbol{B}_{R_{k}, x_{k}}^{(k)}\right) \\
+\frac{R_{k}}{\varepsilon_{k}} \nabla \times \boldsymbol{F}_{k} \text { in } B_{1} .
\end{gathered}
$$

Thus, $\boldsymbol{W}_{k} \in W^{1,2}\left(B_{1}\right)$ is a weak solution to (4.14). Let $0<\sigma<1$. Using the transformation formula, noticing that $\left|\boldsymbol{B}_{R_{k}, x_{k}}^{(k)}\right| \leq M$, the Caccioppoli-type inequality (3.8) with $r=R_{k}$ and $\rho=\sigma R_{k}$ turns into

$$
\left\|\nabla \boldsymbol{W}_{k}\right\|_{2, B_{\sigma}} \leq c(1-\sigma)^{-1}\left((1+M) \mathscr{E}_{k}(1)+\varepsilon_{k} \mathscr{E}_{k}(1)^{2}\right)+\frac{c R_{k}^{-1 / 2}}{\varepsilon_{k}}\|\boldsymbol{F}\|_{2, B_{R_{k}}\left(x_{k}\right)} .
$$

Observing (4.12), and verifying

$$
\frac{R_{k}^{-1 / 2}}{\varepsilon_{k}}\|\boldsymbol{F}\|_{B_{R_{k}}\left(x_{k}\right)} \leq \frac{R_{k}^{(\lambda-1) / 2}}{\varepsilon_{k}}[\boldsymbol{F}]_{\mathcal{M}^{2, \lambda}, K} \leq R_{k}^{(\lambda-1) / 2-\alpha}[\boldsymbol{F}]_{\mathcal{M}^{2, \lambda}, K}
$$

from (4.15), we get

$$
\left\|\nabla \boldsymbol{W}_{k}\right\|_{2, B_{\sigma}} \leq c(1-\sigma)^{-1}(M+1)+c[\boldsymbol{F}]_{\mathcal{M}^{2, \lambda}, K} .
$$

In addition, in view of (4.12) we estimate

$$
\left\|\boldsymbol{W}_{k}\right\|_{4, B_{1}}=\left(\operatorname{mes} B_{1}\right)^{1 / 4} \mathscr{E}_{k}(1) \leq\left(\operatorname{mes} B_{1}\right)^{1 / 4} .
$$


From (4.17) and (4.18) it follows that $\left(\boldsymbol{W}_{k}\right)$ is bounded in $W^{1,2}\left(B_{\sigma}\right)$ for all $0<\sigma<1$ and bounded in $L^{4}\left(B_{1}\right)$. Thus, by means of reflexivity, eventually passing to subsequences, we get $\boldsymbol{W} \in L^{4}\left(B_{1}\right) \cap W_{\text {loc }}^{1,2}\left(B_{1}\right)$ and $\Lambda \in \mathbb{R}^{3}$ such that

$$
\begin{aligned}
\boldsymbol{W}_{k} & \rightarrow \boldsymbol{W} \quad \text { weakly in } \quad L^{4}\left(B_{1}\right) \quad \text { as } \quad k \rightarrow+\infty, \\
\boldsymbol{W}_{k} & \rightarrow \boldsymbol{W} \quad \text { weakly in } W^{1,2}\left(B_{\sigma}\right) \quad \text { as } k \rightarrow+\infty \quad \forall 0<\sigma<1, \\
\boldsymbol{B}_{R_{k}, x_{k}}^{(k)} & \rightarrow \boldsymbol{\Lambda} \quad \text { in } \mathbb{R}^{3} \quad \text { as } \quad k \rightarrow+\infty .
\end{aligned}
$$

On the other hand, by the compactness of the embedding $W^{1,2}\left(B_{\sigma}\right) \hookrightarrow L^{4}\left(B_{\sigma}\right)$ from (4.19) we infer

$$
\boldsymbol{W}_{k} \rightarrow \boldsymbol{W} \quad \text { strongly in } L^{4}\left(B_{\sigma}\right) \quad \text { as } \quad k \rightarrow+\infty \quad \forall 0<\sigma<1 .
$$

Accordingly,

$$
\lim _{k \rightarrow \infty} \mathscr{E}_{k}(\sigma)=\mathscr{E}(\sigma) \quad \forall 0<\sigma<1
$$

where $\mathscr{E}(\sigma)=\left(f_{B_{\sigma}}\left|\boldsymbol{W}-\boldsymbol{W}_{B_{\sigma}}\right|^{4} d y\right)^{1 / 4}$. In particular, by the aid of (4.23) (with $\sigma=\tau)$ from (4.13) we get

$$
\mathscr{E}(\tau) \geq 2 \tau C_{0}\left(1+M^{3}\right)
$$

In view of (4.16) we have

$$
\frac{R_{k}}{\varepsilon_{k}}\left\|\boldsymbol{F}_{k}\right\|_{2, B_{1}}=\frac{R_{k}^{-1 / 2}}{\varepsilon_{k}}\|\boldsymbol{F}\|_{B_{R_{k}}\left(x_{k}\right)} \leq R_{k}^{(\lambda-1) / 2-\alpha}[\boldsymbol{F}]_{\mathcal{M}^{2, \lambda}, K} \rightarrow 0
$$

as $k \rightarrow+\infty$. Therefore, with the help of (4.19), (4.20), (4.21) and (4.22), letting $k \rightarrow+\infty$ in (4.14), we see that $\boldsymbol{W} \in W_{\text {loc }}^{1,2}\left(B_{1}\right) \cap L^{4}\left(B_{1}\right)$ is a weak solution to

$$
-\Delta \boldsymbol{W}=-\nabla \times((\nabla \times \boldsymbol{W}) \times \boldsymbol{\Lambda}) \text { in } B_{1} .
$$

As $|\boldsymbol{\Lambda}| \leq M$ appealing to Lemma 4.1, we find

$$
\mathscr{E}(\tau) \leq \tau C_{0}\left(1+M^{3}\right) \mathscr{E}(1)
$$

On the other hand, by virtue of the lower semi continuity of the norm together with (4.13) and (4.23) we get

$$
\begin{aligned}
\mathscr{E}(1) & \leq \liminf _{k \rightarrow \infty}\left(\mathscr{E}_{k}(1)+\frac{R_{k}^{\alpha}}{\varepsilon_{k}}\right) \leq \frac{1}{2 \tau C_{0}\left(1+M^{3}\right)} \lim _{k \rightarrow \infty} \mathscr{E}_{k}(\tau) \\
& =\frac{1}{2 \tau C_{0}\left(1+M^{3}\right)} \mathscr{E}(\tau) .
\end{aligned}
$$

Estimating the right of (4.26) by the inequality we have just obtained, we see that $\mathscr{E}(\tau) \leq \frac{1}{2} \mathscr{E}(\tau)$ and hence $\mathscr{E}(\tau)=0$, which contradicts to (4.24). Whence, the assumption is not true, and this completes the proof of the Lemma. 


\section{Partial regularity}

The aim of the present section is to prove the partial regularity of a suitable weak solution $\boldsymbol{B} \in W_{\text {loc }}^{1,2}(\Omega)$ to system (3.1), which will lead to the partial regularity of a suitable weak solution to the steady Hall-MHD system. As we will see below, the set $\Sigma(\boldsymbol{B})$ of possible singularities is given by means of

$$
\begin{aligned}
\Sigma(\boldsymbol{B})=\left\{\left.x_{0} \in \Omega\left|\liminf _{r \rightarrow 0^{+}} \frac{1}{r} \int_{B_{r}\left(x_{0}\right)}\right| \nabla \boldsymbol{B}\right|^{2} d x>0\right\} \\
\cup\left\{x_{0} \in \Omega\left|\sup _{r>0}\right| \boldsymbol{B}_{r, x_{0}} \mid=+\infty\right\} .
\end{aligned}
$$

Theorem 5.1. Let $\boldsymbol{F} \in \mathcal{M}_{\text {loc }}^{2, \lambda}(\Omega)$ for some $1<\lambda<3$. Let $\boldsymbol{B} \in W_{\text {loc }}^{1,2}(\Omega)$ be a suitable weak solution to the system (3.1). Then, $\Omega \backslash \Sigma(\boldsymbol{B})$ is an open set, on which $\boldsymbol{B}$ is $\alpha$-Hölder continuous with repect to any exponent $0<\alpha<\frac{\lambda-1}{2}$.

Proof Let $x_{0} \in \Omega \backslash \Sigma(\boldsymbol{B})($ cf. (5.1) $)$. Set $d=\operatorname{dist}\left(x_{0}, \partial \Omega\right)$, and define $K=\overline{B_{d / 2}\left(x_{0}\right)}$. Using Sobolev Poincaré's inequality, we have

$$
\liminf _{r \rightarrow 0^{+}} f_{B_{r}\left(x_{0}\right)}\left|\boldsymbol{B}-\boldsymbol{B}_{r, x_{0}}\right|^{4} d x \leq c \liminf _{r \rightarrow 0^{+}}\left(\frac{1}{r} \int_{B_{r}\left(x_{0}\right)}|\nabla \boldsymbol{B}|^{2} d x\right)^{2}=0 .
$$

We set

$$
M:=\sup _{0<r<d / 2}\left|\boldsymbol{B}_{r, x_{0}}\right|+3<+\infty
$$

Take $\tau>0$ such that

$$
2 \tau^{1-\alpha} C_{0}\left(1+M^{3}\right) \leq \frac{1}{2}, \quad \tau^{\alpha} \leq \frac{1}{2} .
$$

Let $\varepsilon_{0}=\varepsilon_{0}(\tau, M, K, \alpha)$ and $R_{0}=R_{0}(\tau, M, K, \alpha)$ denote the numbers according to Lemma 4.2. Then, we choose $0<R_{1} \leq R_{0}$ such that

$$
E\left(R_{1}, x_{0}\right)+2 R_{1}^{\alpha}<\min \left\{\varepsilon_{0}, \varepsilon_{1}\right\}
$$

where $\varepsilon_{1}>0$ fulfills

$$
2 \tau^{-3} \varepsilon_{1} \leq 1
$$

By the absolutely continuity of the Lebesgue integral there exists $0<\delta<\frac{d}{2}$ such that for all $y \in \overline{B_{\delta}\left(x_{0}\right)}$

$$
\begin{aligned}
& E\left(y, R_{1}\right)+2 R_{1}^{\alpha} \leq \min \left\{\varepsilon_{0}, \varepsilon_{1}\right\} \\
& \left|\boldsymbol{B}_{R_{1}, y}\right| \leq \sup _{0<r<d / 2}\left|\boldsymbol{B}_{r, x_{0}}\right|+1=M-2
\end{aligned}
$$


Fix $y \in \overline{B_{\delta}\left(x_{0}\right)}$. We claim that for every $j \in \mathbb{N} \cup\{0\}$ there holds

$$
\begin{aligned}
E\left(\tau^{j} R_{1}, y\right) & \leq 2^{-j} \tau^{\alpha j} E\left(R_{1}, y\right)+\left(1-2^{-j}\right) \tau^{\alpha j} R_{1}^{\alpha}, \\
\left|\boldsymbol{B}_{\tau^{j} R_{1}, y}\right| & \leq M-2^{-j+1} .
\end{aligned}
$$

Clearly, for $j=0$, (5.7) is trivially fulfilled, while (5.8) holds in view of (5.6).

Now, assume (5.7) and (5.8) are fulfilled for $j \in N \cup\{0\}$. Then (5.7) along with (5.3) immediately implies

$$
E\left(\tau^{j} R_{1}, y\right)+\tau^{\alpha j} R_{1}^{\alpha} \leq \tau^{\alpha j}\left(E\left(R_{1}, y\right)+2 R_{1}^{\alpha}\right) \leq \tau^{\alpha j} \min \left\{\varepsilon_{0}, \varepsilon_{1}\right\} .
$$

Now, both (5.8) and (5.9) imply

$$
E\left(\tau^{j} R_{1}, y\right)+\tau^{\alpha j} R_{1}^{\alpha} \leq \varepsilon_{0}, \quad\left|\boldsymbol{B}_{\tau^{j} R_{1} . y}\right| \leq M
$$

Thus, we are in a position to apply Lemma 4.2 with $R=\tau^{j} R_{1}$. This together with (5.2) gives

$$
\begin{aligned}
E\left(\tau^{j+1} R_{1}, y\right) & \leq 2 \tau C_{0}\left(1+M^{3}\right)\left(E\left(\tau^{j} R_{1}, y\right)+\tau^{\alpha j} R_{1}^{\alpha}\right) \\
& \leq \frac{1}{2} \tau^{\alpha} E\left(\tau^{j} R_{1}, y\right)+\frac{1}{2} \tau^{\alpha(j+1)} R_{1}^{\alpha} \\
& \leq 2^{-(j+1)} \tau^{\alpha(j+1)} E\left(R_{1}, y\right)+\left(1-2^{-(j+1)}\right) \tau^{\alpha(j+1)} R_{1}^{\alpha} .
\end{aligned}
$$

This proves (5.7) for $j+1$.

It remains to show (5.8) for $j+1$. First, from (5.7) along with (5.5) we infer

$$
E\left(\tau^{j} R_{1}, y\right) \leq \tau^{\alpha j}\left(E\left(R_{1}, y\right)+R_{1}^{\alpha}\right) \leq \tau^{\alpha j} \varepsilon_{1}
$$

Using triangle inequality and Jensen's inequality, we find

$$
\begin{aligned}
\left|\boldsymbol{B}_{\tau^{j+1} R_{1}, y}\right| & \leq\left|\boldsymbol{B}_{\tau^{j} R_{1}, y}\right|+\left|\boldsymbol{B}_{\tau^{j+1} R_{1}, y}-\boldsymbol{B}_{\tau^{j} R_{1}, y}\right| \\
& \leq\left|\boldsymbol{B}_{\tau^{j} R_{1}, y}\right|+2 \tau^{-3} E\left(\tau^{j} R_{1}, y\right) .
\end{aligned}
$$

Estimating the first member on the right by using (5.8) and the second one by the aid of (5.11) together with (5.3) and (5.4), we obtain

$$
\begin{aligned}
\left|\boldsymbol{B}_{\tau^{j+1} R_{1}, y}\right| & \leq M-2^{-j+1}+2 \tau^{-3} \tau^{\alpha j} \varepsilon_{1} \\
& \leq M-2^{-j+1}+2^{-j}=M-2^{-j} .
\end{aligned}
$$

This completes the proof of (5.8) for $j+1$. Whence, the claim.

From (5.7) we get a constant $C_{1}>0$ such that

$$
\left(f_{B_{r}(y)}\left|\boldsymbol{B}-\boldsymbol{B}_{r, y}\right|^{4} d x\right)^{1 / 4} \leq C_{1} r^{\alpha} \quad \forall 0<r<\frac{d}{2}, \quad \forall y \in \overline{B_{\delta}\left(x_{0}\right)} .
$$

Thus, by the well-known equivalence of the Campanato space and the Hölder space(see e.g. [3] or Theorem 1.3 of [11]) we conclude

$$
\left.\boldsymbol{B}\right|_{\overline{B_{\delta}\left(x_{0}\right)}} \in C^{\alpha}\left(\overline{B_{\delta}\left(x_{0}\right)}\right) .
$$


Finally, we shall verify that $B_{\delta}\left(x_{0}\right) \subset \Omega \backslash \Sigma(\boldsymbol{B})$. Let $y \in B_{\delta}\left(x_{0}\right)$ be arbitrarily chosen. Firstly, notice that $\sup _{0<r<d / 2}\left|\boldsymbol{B}_{r, y}\right|<+\infty$ (see (5.12) ). Secondly, from the Cacciopploli-type inequality (3.8) with $0<r<\frac{d}{2}$ and $\rho=\frac{r}{2}$ replacing $x_{0}$ by $y$ therein we deduce

$$
\begin{aligned}
& \left(\frac{1}{r} \int_{B_{r / 2}(y)}|\nabla \boldsymbol{B}|^{2} d x\right)^{1 / 2} \\
& \quad \leq c\left\{\left(1+\left|\boldsymbol{B}_{r, y}\right|\right) E(r, y)+E(r, y)^{2}\right\}+c r^{(\lambda-1) / 2}[\boldsymbol{F}]_{\mathcal{M}^{2, \lambda}, K} .
\end{aligned}
$$

As $\lim _{r \rightarrow 0^{+}} E(r, y)=0$ and $\lambda>1$ the right-hand side of the above inequaliy tends to zero as $r \rightarrow 0^{+}$. Hence, $y \in \Omega \backslash \Sigma(\boldsymbol{B})$. This completes the proof of the theorem.

Remark 5.2. By the result of [11, Chap. IV, 2.] there holds

$$
\mathcal{H}^{\beta}(\Sigma(\boldsymbol{B}))=0 \quad \forall \beta>1,
$$

which implies that $\Sigma(\boldsymbol{B})$ has Hausdorff dimension at most one. We don't know, however, whether the one-dimensional Hausdorff measure of $\Sigma(\boldsymbol{B})$ is finite.

As a consequence of Theorem 5.1 we get the following partial regularity result for the steady Hall-MHD system.

Theorem 5.3. Let $\boldsymbol{f} \in L^{6 / 5} \cap L_{\text {loc }}^{2}$ and $\boldsymbol{g} \in L^{2} \cap \mathcal{M}_{\text {loc }}^{2, \lambda}$ for some $1<\lambda \leq 2$. Let $(\boldsymbol{u}, p, \boldsymbol{B})$ be a weak solution to the Hall-MHD system such that $\boldsymbol{B}$ satisfies the local energy inequality (3.3) with $\boldsymbol{F}=-\boldsymbol{B} \times \boldsymbol{u}+\boldsymbol{g}$. Then, for $\Sigma(\boldsymbol{B})$ defined by (5.1) we have that $\mathbb{R}^{3} \backslash \Sigma(\boldsymbol{B})$ is an open set such that $\boldsymbol{B}$ is $\alpha$-Hölder continuous on $\mathbb{R}^{3} \backslash \Sigma(\boldsymbol{B})$ for any $0<\alpha<\frac{\lambda-1}{2}$.

Proof Arguing similarly as in the proof of Theorem 2.3, from $\boldsymbol{f}, \boldsymbol{g} \in L_{\text {loc }}^{2}$ we deduce that $\boldsymbol{V}=\boldsymbol{B}+\boldsymbol{\omega} \in \boldsymbol{W}_{\text {loc }}^{1,2}$. As $\boldsymbol{B} \in W^{1,2}$ we see that $\boldsymbol{\omega} \in W_{\text {loc }}^{1,2}$, and hence $\boldsymbol{u} \in W_{\text {loc }}^{2,2}$. By means of Sobolev's embedding theorem we find $\boldsymbol{u} \in L_{\text {loc. }}^{\infty}$ This shows that $\boldsymbol{B} \times \boldsymbol{u} \in$ $L_{\text {loc }}^{6} \subset \mathcal{M}_{\text {loc }}^{2,2}$. Consequently, $\boldsymbol{F}=-\boldsymbol{B} \times \boldsymbol{u}+\boldsymbol{g} \in \mathcal{M}_{\mathrm{loc}}^{2, \lambda}$. The assertion of the theorem is now an immediate consequence of Theorem 5.1.

\section{Higher regularity}

In Section 5 we have proved the partial Hölder regularity of a suitable weak solution $(\boldsymbol{u}, p, \boldsymbol{B})$ of the Hall-MHD system for $\boldsymbol{f}$ and $\boldsymbol{g}$ being sufficiently regular. The aim of the present section is to show that if both $\boldsymbol{f}$ and $\boldsymbol{g}$ are smooth, then $(\boldsymbol{u}, p, \boldsymbol{B})$ is smooth in $\mathbb{R}^{3} \backslash \Sigma(\boldsymbol{B})$. To prove this we first shall establish a regularity result for the following linerized problem.

Let $\Omega \subset \mathbb{R}^{3}$ be an open set, and let $\boldsymbol{B} \in C(\Omega)$. We consider the linear system

$$
-\Delta \boldsymbol{A}=-\nabla \times((\nabla \times \boldsymbol{A}) \times \boldsymbol{B})+\nabla \times \boldsymbol{F} \text { in } \Omega .
$$

We have the following regularity result. 
Theorem 6.1. Let $\boldsymbol{F} \in \mathcal{M}_{\text {loc }}^{2, \lambda}(\Omega)$ for some $1<\lambda<3$. Let $\boldsymbol{A} \in W_{\text {loc }}^{1,2}(\Omega)$ be a weak solution to (6.1). Then

$$
\boldsymbol{A} \in C^{\alpha}(\Omega) \quad \text { with } \quad \alpha=\frac{\lambda-1}{2} .
$$

Proof Let $x_{0} \in \Omega$. Set $d=\operatorname{dist}\left(x_{0}, \partial \Omega\right)$. As $\boldsymbol{B}$ is continuous, we get

$$
M:=\max _{y \in \overline{B_{d / 2}\left(x_{0}\right)}}|\boldsymbol{B}(y)|<+\infty .
$$

Let $y \in B_{d / 4}\left(x_{0}\right)$ be fixed. For the sake of notational simplicity in what follows we use the notation

$$
S(R, y)=\left(\frac{1}{R} \int_{B_{R}(y)}|\nabla \boldsymbol{A}|^{2} d x\right)^{1 / 2}, \quad 0<R \leq \frac{d}{4} .
$$

Furthermore, by $\operatorname{osc}\left(f ; x_{0}, R\right)$ we denote the oscillation of a continuous function $f$ over $B_{R}\left(x_{0}\right)$, which equals the supremum of $|f(x)-f(y)|$ taken over all $x, y \in B_{R}\left(x_{0}\right)$. As $\boldsymbol{B}$ is continuous we may choose $0<R_{0}<\frac{d}{4}$ such that

$$
\sup _{B_{R}(y)}\left|\boldsymbol{B}-\boldsymbol{B}_{R, y}\right| \leq \operatorname{osc}\left(\boldsymbol{B} ; x_{0}, 2 R\right) \leq \frac{1}{2} \quad \forall 0<R \leq R_{0} .
$$

Next, for $0<R \leq R_{0}$ let $\boldsymbol{Z} \in W^{1,2}\left(B_{R}(y)\right)$ denote the unique weak solution to

$$
\begin{aligned}
-\Delta \boldsymbol{Z}= & -\nabla \times\left((\nabla \times \boldsymbol{Z}) \times \boldsymbol{B}_{R, y}\right) \\
& -\nabla \times\left((\nabla \times \boldsymbol{A}) \times\left(\boldsymbol{B}-\boldsymbol{B}_{R, y}\right)\right)+\nabla \times \boldsymbol{F} \text { in } B_{R}(y), \\
\boldsymbol{Z}=\mathbf{0} \text { on } \partial B_{R}(y) . &
\end{aligned}
$$

It can be easily checked that

$$
\begin{aligned}
R^{-1 / 2}\|\nabla \boldsymbol{Z}\|_{2, B_{R}(y)} & \leq 4 \operatorname{osc}\left(\boldsymbol{B} ; x_{0}, 2 R\right) R^{-1 / 2}\|\nabla \boldsymbol{A}\|_{2, B_{R}(y)}+2 R^{-1 / 2}\|\boldsymbol{F}\|_{2, B_{R}(y)} \\
& \leq 4 \operatorname{osc}\left(\boldsymbol{B} ; x_{0}, 2 R\right) S(R, y)+2 R^{(\lambda-1) / 2}[\boldsymbol{F}]_{\mathcal{M}^{2, \lambda}, B_{d / 2}\left(x_{0}\right)} .
\end{aligned}
$$

Setting $\boldsymbol{W}=\boldsymbol{A}-\boldsymbol{Z}$ and $\boldsymbol{\Lambda}=\boldsymbol{A}_{R, y}$, it follows that $\boldsymbol{W} \in W^{1,2}\left(B_{R}(y)\right)$ is a weak solution to (4.1) in $B_{R}(y)$. Analogeously as Lemma 4.1 one shows that get

$$
\left(\frac{1}{\tau R} \int_{B_{\tau R}(y)}|\nabla \boldsymbol{W}|^{2} d x\right)^{1 / 2} \leq C_{0} \tau\left(1+M^{2}\right)\left(\frac{1}{R} \int_{B_{R}(y)}|\nabla \boldsymbol{W}|^{2} d x\right)^{1 / 2}
$$

for all $0<\tau<1$.

Next, let $\frac{\lambda-1}{2}<\beta<1$ be fixed. Take $0<\tau<\frac{1}{2}$ such that

$$
C_{0} \tau^{1-\beta}\left(1+M^{2}\right) \leq \frac{1}{2} .
$$


Thus, using triangle inequality along with (6.5), (6.6) and (6.7) , we get

$$
\begin{aligned}
S(\tau R, y) & \leq\left(\frac{1}{\tau R} \int_{B_{\tau R}(y)}\left|\nabla \boldsymbol{W}_{\tau R, y}\right|^{2} d x\right)^{1 / 2}+\tau^{-1 / 2} R^{-1 / 2}\|\nabla \boldsymbol{Z}\|_{2, R} \\
& \leq C_{0} \tau\left(1+M^{2}\right) S(R, y)+\left(1+\tau^{-1 / 2}\right) R^{-1 / 2}\|\nabla \boldsymbol{Z}\|_{2, R} \\
& \leq \frac{1}{2} \tau^{\beta} S(R, y)+4\left(1+\tau^{-1 / 2}\right) \operatorname{osc}\left(\boldsymbol{B} ; x_{0}, 2 R\right) S(R, y)+C_{1} R^{\alpha}
\end{aligned}
$$

where

$$
C_{1}=2\left(1+\tau^{-1 / 2}\right)[\boldsymbol{F}]_{\mathcal{M}^{2, \lambda}, B_{d / 2}\left(x_{0}\right)}, \quad \alpha=\frac{\lambda-1}{2} .
$$

Take $0<R_{1} \leq R_{0}$ such that $4\left(1+\tau^{-1 / 2}\right) \operatorname{osc}\left(\boldsymbol{B} ; x_{0}, 2 R_{1}\right) \leq \frac{\tau^{\beta}}{2}$. Then from the estimate above we deduce

$$
S(\tau R, y) \leq \tau^{\beta} S(R, y)+C_{1} R^{\alpha} \quad \forall 0<R \leq R_{1}
$$

By using a routine iteration argument, we infer from (6.9) that that

$$
\begin{aligned}
S\left(\tau^{k} R_{1}, y\right) & \leq \tau^{\beta} S\left(\tau^{k-1} R_{1}\right)+C_{1} \tau^{\alpha k} R_{1}^{\alpha} \\
& \leq \tau^{k \beta} S\left(\tau^{k-2} R_{1}\right)+C_{1} \tau^{\alpha k}\left(1+\tau^{\beta-\alpha}+\ldots+\tau^{(\beta-\alpha)(k-1)}\right) R_{1}^{\alpha} \\
& \leq \tau^{k \alpha}\left(1+C_{1} R_{1}^{\alpha} \frac{1}{1-\tau^{\beta-\alpha}}\right) .
\end{aligned}
$$

Thus, there exists a constant $C_{2}>0$ such that

$$
S(R, y) \leq C_{2} R^{\alpha} \quad \forall 0<R \leq \frac{d}{4}, \quad y \in B_{d / 4}\left(x_{0}\right)
$$

By the aid of the Poincaré inequality from (6.10) we obtain

$$
\left(f_{B_{R}}\left|\boldsymbol{A}-\boldsymbol{A}_{R, y}\right|^{2} d x\right)^{1 / 2} \leq c R^{\alpha} \quad \forall 0<R \leq \frac{1}{4}, \quad y \in B_{d / 4}\left(x_{0}\right),
$$

which leads to the desired Hölder regularity of $\boldsymbol{A}$.

We are now in a position to prove the higher regularity for a continuous weak solution $(\boldsymbol{u}, p, \boldsymbol{B})$ to the steady Hall-MHD system. More precisely, we have the following

Theorem 6.2. For $\boldsymbol{f} \in L^{6 / 5}$ and $\boldsymbol{g} \in L^{2}$ let $(\boldsymbol{u}, p, \boldsymbol{B})$ be a weak solution to the steady Hall-MHD system. Let $\Omega \subset \mathbb{R}^{3}$ be an open set such that $\boldsymbol{B}$ is continuous in $\Omega$ and $\boldsymbol{f}, \boldsymbol{g} \in C^{k}(\Omega) \quad(k \in \mathbb{N} \cup\{0\})$. Then $\boldsymbol{B}, \boldsymbol{\omega} \in C^{k, \alpha}(\Omega)$ for all $0<\alpha<1$.

Proof First, let us consider the case $k=0$. As $\boldsymbol{f}, \boldsymbol{g} \in L_{\mathrm{loc}}^{\infty}(\Omega)$ by virtue of Theorem 2.3 and Sobolev's embedding theorem we get

$$
\boldsymbol{V} \in W_{\mathrm{loc}}^{1, q}(\Omega) \quad \forall 1 \leq q<+\infty, \quad \boldsymbol{V} \in C_{\mathrm{loc}}^{\alpha}(\Omega) \quad \forall 0<\alpha<1 .
$$


With help of Sobolev's embedding theorem we see that $-\boldsymbol{B} \times \boldsymbol{u}+\boldsymbol{g} \in \mathcal{M}_{\text {loc }}^{2, \lambda}$ for all $0<\lambda<3$. Hence, from Theorem 6.1 with $\boldsymbol{A}=\boldsymbol{B}$ we immediately get $\boldsymbol{B} \in C_{\mathrm{loc}}^{\alpha}(\Omega)$ for all $0<\alpha<1$. As $\boldsymbol{\omega}=\boldsymbol{V}-\boldsymbol{B}$ we infer $\boldsymbol{\omega} \in C_{\mathrm{loc}}^{\alpha}(\Omega)$ and since $\nabla \cdot \boldsymbol{u}=0$ it follows $\boldsymbol{u} \in C_{\mathrm{loc}}^{1, \alpha}(\Omega)$. This completes the proof of the assertion in case $k=0$.

Suppose $\boldsymbol{f}, \boldsymbol{g} \in C_{\mathrm{loc}}^{k}(\Omega)$ for some $k \in \mathbb{N}$. From the proof above we immediately get $\boldsymbol{B}, \boldsymbol{\omega} \in C_{\mathrm{loc}}^{\alpha}(\Omega)$ for all $0<\alpha<1$. Now, assume that $\boldsymbol{B}, \boldsymbol{\omega} \in C_{\mathrm{loc}}^{j-1, \alpha}(\Omega) \cap W_{\text {loc }}^{j, 2}(\Omega)$ for all $0<\alpha<1$ for some $j \in\{1, \ldots, k-1\}$. Let $\nu \in \mathbb{N}^{3}$ be a multi-index with $|\nu|=j-1$. Define $\boldsymbol{A}=D^{\nu} \boldsymbol{B}$ in $\Omega$. Applying $D^{\nu}$ to both sides of (2.2), we are led to

$$
\begin{aligned}
-\Delta \boldsymbol{A}= & -\nabla \times((\nabla \times \boldsymbol{A}) \times \boldsymbol{B}) \\
& -\sum_{|\mu| \leq j-2, \mu \leq \nu} \nabla \times\left(\left(\nabla \times D^{\mu} \boldsymbol{B}\right) \times D^{\nu-\mu} \boldsymbol{B}\right)+\nabla \times D^{\nu}(\boldsymbol{B} \times \boldsymbol{u}+\boldsymbol{g}) \\
= & -\nabla \times((\nabla \times \boldsymbol{A}) \times \boldsymbol{B})+\nabla \times \boldsymbol{G}
\end{aligned}
$$

in $\Omega$. By our assumption, we have $\boldsymbol{G} \in C_{\mathrm{loc}}^{\alpha}(\Omega)$ for all $0<\alpha<1$. Applying the method of differences, we see that $\boldsymbol{A} \in W_{\text {loc }}^{1+\theta, 2}(\Omega)$ for every $0<\theta<1$. Consequently, $\boldsymbol{B} \in W_{\text {loc }}^{j+\theta, 2}(\Omega)$ for all $0<\theta<1$. By virtue of Sobolev's embedding theorem it follows that

$$
\boldsymbol{B} \in W_{\mathrm{loc}}^{j, q}(\Omega) \quad \forall 1 \leq q<6 .
$$

This shows that the $\nabla \times \boldsymbol{G} \in L_{\text {loc }}^{2}(\Omega)$. Therefore, we are able to perform the method of difference quotient which yields $\boldsymbol{A} \in W_{\text {loc }}^{2,2}(\Omega)$. Recalling our assumption, having $\boldsymbol{A} \in C_{\text {loc }}^{\alpha}(\Omega)$ for all $0<\alpha<1$ by the interpolation inequality due to Kufner and Wannebo [13], we obtain $\boldsymbol{A} \in W_{\text {loc }}^{1, q}(\Omega)$ for all $1 \leq q<+\infty$. This proves that

$$
\boldsymbol{B} \in W_{\mathrm{loc}}^{j+1,2}(\Omega) \cap \bigcap_{1 \leq q<\infty} W_{\mathrm{loc}}^{j, q}(\Omega) .
$$

Repeating the above argument with $|\nu|=j$ and $\boldsymbol{A}=D^{\nu} \boldsymbol{B}$, we see that $\boldsymbol{A} \in W_{\text {loc }}^{1,2}(\Omega)$ is a weak solution to

$$
-\Delta \boldsymbol{A}=-\nabla \times(\nabla \times \boldsymbol{A}) \times \boldsymbol{B})+\nabla \times \boldsymbol{G}
$$

Thanks to (6.15) we have $\boldsymbol{G} \in \mathcal{M}_{\mathrm{loc}}^{2, \lambda}(\Omega)$ for all $0<\lambda<3$, so that Theorem 6.1 yields that $\boldsymbol{A} \in C_{\mathrm{loc}}^{\alpha}(\Omega)$ for all $0<\alpha<1$, and that implies

$$
\boldsymbol{B} \in C_{\mathrm{loc}}^{j, \alpha}(\Omega) \quad \forall 0<\alpha<1 .
$$

Furthermore, according to our assumption we have $\boldsymbol{V} \in W_{\text {loc }}^{j-1, q}(\Omega)$ for all $1 \leq$ $q<+\infty$. Consequently, $-\boldsymbol{V} \times \boldsymbol{u}+\boldsymbol{f}+\boldsymbol{g} \in W_{\mathrm{loc}}^{j-1, q}(\Omega)$ for all $1 \leq q<+\infty$. Hence using the Calderón-Zygmund inequality, from (2.6) we deduce that $\boldsymbol{V} \in W_{\text {loc }}^{j, q}(\Omega)$ for all $1 \leq q<+\infty$. In particular, $-\boldsymbol{V} \times \boldsymbol{u}+\boldsymbol{f}+\boldsymbol{g} \in W_{\mathrm{loc}}^{j, q}(\Omega)$ for all $1 \leq q<+\infty$. Once, more employing Calderón-Zygmund's inequality, we find $\boldsymbol{V} \in W_{\text {loc }}^{j+1, q}(\Omega)$ for all $1 \leq q<+\infty$, and with the help of Sobolev's embedding theoren we get $\boldsymbol{V} \in C_{\text {loc }}^{j, \alpha}(\Omega)$ 
for all $0<\alpha<1$. Finally, recalling $\boldsymbol{\omega}=\boldsymbol{V}-\boldsymbol{B}$ in view of (6.15) and (6.17), we conclude

$$
\boldsymbol{\omega} \in W_{\mathrm{loc}}^{j+1,2}(\Omega) \cap \bigcap_{0<\alpha<1} C_{\mathrm{loc}}^{j, \alpha}(\Omega)
$$

The desired regularity now follows from above by induction over $j=0, \ldots, k$.

\section{Direct method and compacteness of the singular set}

In this section we prove that a suitable weak solution becomes regular outside a sufficiently large ball, which is due to the decay property

$$
\lim _{R \rightarrow+\infty} \int_{\{|x|>R\}}|\boldsymbol{B}|^{6} d x=0 .
$$

First let us state an alternative Caccioppoli-type inequality for the system (3.1).

Lemma 7.1. Let $\boldsymbol{F} \in L^{2}$ and let $\boldsymbol{B} \in \dot{W}^{1,2}$ be a suitable weak solution to the system (3.1) in $\mathbb{R}^{3}$. Then for every Ball $B_{r}=B_{r}\left(x_{0}\right)$ and $0<\rho<r$ there holds

$$
\begin{aligned}
& \frac{1}{r} \int_{B_{\rho}}|\nabla \boldsymbol{B}|^{2} d x \\
& \leq \frac{c r^{2}}{(r-\rho)^{2}}\left\{1+\left(f_{B_{r}}|\boldsymbol{B}|^{6} d x\right)^{1 / 3}\right\}\left(f_{B_{r}}\left|\boldsymbol{B}-\boldsymbol{B}_{r, x_{0}}\right|^{3} d x\right)^{2 / 3} \\
& \quad+\frac{c}{r}\|\boldsymbol{F}\|_{2, B_{r}}^{2},
\end{aligned}
$$

where $c=$ const $>0$ denotes a universal constant.

Proof This can be easily achieved by estimating the integral $J$ on the right-hand side of (3.6) by using Hölder's inequality and Young's inequality as follows

$$
\begin{aligned}
J & \leq \frac{c}{(r-\rho)^{2}} \int_{B_{r}}|\boldsymbol{B}|^{2}\left|\boldsymbol{B}-\boldsymbol{B}_{r, x_{0}}\right|^{2} d x+\frac{1}{2} \int_{B_{r}} \zeta^{2}|\nabla \boldsymbol{B}|^{2} d x \\
& \leq \frac{c r^{3}}{(r-\rho)^{2}}\left(f_{B_{r}}|\boldsymbol{B}|^{6} d x\right)^{1 / 3}\left(f_{B_{r}} \boldsymbol{B}-\left.\boldsymbol{B}_{r, x_{0}}\right|^{3} d x\right)^{2 / 3}+\frac{1}{2} \int_{B_{r}} \zeta^{2}|\nabla \boldsymbol{B}|^{2} d x .
\end{aligned}
$$
lowing

Using the well-known properties of harmonic functions, one easily verifies the fol- 
Lemma 7.2. Let $\boldsymbol{W} \in W^{1,3 / 2}\left(B_{R / 2}\left(x_{0}\right)\right)$ be harmonic in $B_{R / 2}\left(x_{0}\right)$. Then, there exists an absolute constant $C_{0}$ such that for all $0<\tau<\frac{1}{2}$

$$
\left(f_{B_{\tau R}}\left|\boldsymbol{W}-\boldsymbol{W}_{\tau R, x_{0}}\right|^{3} d x\right)^{1 / 3} \leq C_{0} \tau\left(f_{B_{R / 2}}\left|\boldsymbol{W}-\boldsymbol{W}_{R / 2, x_{0}}\right|^{3} d x\right)^{1 / 3} .
$$

In what follows, let $\boldsymbol{F} \in \mathcal{M}^{2, \lambda}$ for some $1<\lambda<3$, i. e.

$$
[\boldsymbol{F}]_{\mathcal{M}^{2, \lambda}}^{2}=\sup \left\{r^{-\lambda} \int_{B_{r}\left(x_{0}\right)}|\boldsymbol{F}|^{2} d x \mid x_{0} \in \mathbb{R}^{3}, 0<r \leq 1\right\}<+\infty .
$$

Clearly

$$
R^{-1 / 2}\|\boldsymbol{F}\|_{2, B_{R}} \leq \gamma_{0} R^{\alpha} \quad \forall 0<R \leq 1,
$$

where

$$
\alpha=\frac{\lambda-1}{2}, \quad \gamma_{0}=[\boldsymbol{F}]_{\mathcal{M}^{2, \lambda}} .
$$

Furthermore, define

$$
\begin{aligned}
S\left(r, x_{0}\right) & =\left(\frac{1}{r} \int_{B_{r}\left(x_{0}\right)}|\nabla \boldsymbol{B}|^{2} d x\right)^{1 / 2}, \\
E\left(r, x_{0}\right) & =\left(f_{B_{r}\left(x_{0}\right)}\left|\boldsymbol{B}-\boldsymbol{B}_{r, x_{0}}\right|^{3} d x\right)^{1 / 3}, \\
M\left(r, x_{0}\right) & =\left(f_{B_{r}\left(x_{0}\right)}|\boldsymbol{B}|^{6} d x\right)^{1 / 6}, \quad 0<r<+\infty, x_{0} \in \mathbb{R}^{3} .
\end{aligned}
$$

Fix $x_{0} \in \mathbb{R}^{3}$ and $0<R \leq 1$. Assume that $M\left(R, x_{0}\right) \leq 1$. Then, from (7.2) with $r=R$ and $\rho=\frac{R}{2}$ along with (7.4) we deduce

$$
S\left(R / 2, x_{0}\right) \leq c\left(E\left(R, x_{0}\right)+\gamma_{0} R^{\alpha}\right),
$$

where $c>0$ denotes an absolute constant.

Let $\alpha<\beta<1$ be fixed. Take $0<\tau<\frac{1}{4}$ such that

$$
2 C_{0} \tau^{1-\beta} \leq \frac{1}{2}, \quad \tau^{\beta-\alpha} \leq \frac{1}{2}, \quad \tau^{\alpha} \leq \frac{1}{2} .
$$

Let $\boldsymbol{Z} \in W^{1,3 / 2}\left(B_{R / 2}\left(x_{0}\right)\right)$ denote a weak solution to

$$
\begin{aligned}
-\Delta \boldsymbol{Z} & =-\nabla \times((\nabla \times \boldsymbol{B}) \times \boldsymbol{B})+\nabla \times \boldsymbol{F} \text { in } B_{R / 2}\left(x_{0}\right), \\
\boldsymbol{Z} & =\mathbf{0} \quad \text { on } \quad \partial B_{R / 2}\left(x_{0}\right) .
\end{aligned}
$$


By the well-known $L^{p}$-theory of the Laplace equation we get

$$
\begin{aligned}
R^{-1}\|\nabla \boldsymbol{Z}\|_{3 / 2, B_{R / 2}\left(x_{0}\right)} & \leq c R^{-1}\|(\nabla \times \boldsymbol{B}) \times \boldsymbol{B}\|_{3 / 2, B_{R / 2}\left(x_{0}\right)}+c R^{-1}\|\boldsymbol{F}\|_{3 / 2, B_{R}\left(x_{0}\right)} \\
& \leq c M\left(R, x_{0}\right) S\left(R / 2, x_{0}\right)+c \gamma_{0} R \alpha .
\end{aligned}
$$

Esimating the left hand side from below by using Sobolev-Poincaré's inequality and the right hand side from above by the aid of (7.5), recalling that $M\left(R, x_{0}\right) \leq 1$, we are led to

$$
\left(f_{B_{R / 2}\left(x_{0}\right)}|\boldsymbol{Z}|^{3} d x\right)^{1 / 3} \leq C_{1}\left(M\left(R, x_{0}\right) E\left(R, x_{0}\right)+\gamma_{0} R^{\alpha}\right),
$$

where $C_{1}>1$ stands for an absolute constant.

Next, we ssume that

$$
3 \tau^{-1} C_{1} M\left(R, x_{0}\right) \leq \frac{1}{2} \tau^{\beta} .
$$

We note here that (7.11) yields $M\left(R, x_{0}\right) \leq 1$ and thus (7.5) remains true. We make use of triangle inequality, then apply (17.3) (note that $\boldsymbol{W}$ is harmonic). This together with (7.10) and (7.6) gives

$$
\begin{aligned}
E\left(\tau R, x_{0}\right) & \leq\left(f_{B_{\tau R}\left(x_{0}\right)}\left|\boldsymbol{W}-\boldsymbol{W}_{\tau R, x_{0}}\right|^{3} d x\right)^{1 / 3}+3 \tau^{-1}\left(f_{B_{R / 2}\left(x_{0}\right)}|\boldsymbol{Z}|^{3} d x\right)^{1 / 3} \\
& \leq 2 C_{0} \tau E\left(R, x_{0}\right)+3 \tau^{-1}\left(f_{B_{R / 2}\left(x_{0}\right)}|\boldsymbol{Z}|^{3} d x\right)^{1 / 3} \\
& \leq \frac{1}{2} \tau^{\beta} E\left(R, x_{0}\right)+3 \tau^{-1} C_{1} M\left(R, x_{0}\right) E\left(R, x_{0}\right)+3 \tau^{-1} C_{1} \gamma_{0} R^{\alpha}
\end{aligned}
$$

and observing (7.11), we therefore obtain

$$
E\left(\tau R, x_{0}\right) \leq \tau^{\beta} E\left(R, x_{0}\right)+C_{2} \gamma_{0} R^{\alpha},
$$

where

$$
C_{2}=3 \tau^{-1} C_{1}
$$

Next, we shall estimate $\left|M\left(\tau R, x_{0}\right)\right|$. By using triangle inequality and SobolevPoincaré inequality it follows that

$$
\begin{aligned}
M\left(\tau R, x_{0}\right) & \leq\left|\boldsymbol{B}_{\tau R, x_{0}}\right|+c \tau^{-1 / 2} S\left(R / 2, x_{0}\right) \\
& \leq M\left(R, x_{0}\right)+c \tau^{-1 / 2} S\left(R / 2, x_{0}\right)+2 \tau^{-1} E\left(R, x_{0}\right) \\
& \leq M\left(R, x_{0}\right)+C_{3}\left(E\left(R, x_{0}\right)+\gamma_{0} R^{\alpha}\right)
\end{aligned}
$$

with a constant $C_{3}>0$ depending on $\tau$ only.

Let $0<M_{0} \leq 1$ such that

$$
3 \tau^{-1} C_{1} M_{0}=\frac{1}{2} \tau^{\beta} .
$$


Let $0<R_{1} \leq 1$ chosen so that

$$
2 C_{3}\left(2 C_{2} \tau^{-\alpha}+1\right) \gamma_{0} R_{1}^{\alpha} \leq \frac{M_{0}}{2}
$$

Since $\boldsymbol{B} \in L^{6}$, there exists $0<\rho_{0}<+\infty$ such that

$$
M\left(R_{1}, x_{0}\right)+2 C_{3} E\left(R_{1}, x_{0}\right) \leq\left(1+4 C_{3}\right) M\left(R_{1}, x_{0}\right) \leq \frac{M_{0}}{2} \quad \forall\left|x_{0}\right| \geq \rho_{0} .
$$

Let $x_{0} \in \mathbb{R}^{n},\left|x_{0}\right| \geq \rho_{0}$. We claim that for every $k \in \mathbb{N} \cup\{0\}$

$$
\begin{aligned}
E\left(\tau^{k} R_{1}, x_{0}\right) & \leq \tau^{\beta k} E\left(R_{1}, x_{0}\right)+2\left(1-2^{-k}\right) \tau^{\alpha(k-1)} C_{2} \gamma_{0} R_{1}^{\alpha}, \\
M\left(\tau^{k} R_{1}, x_{0}\right) & \leq M\left(R_{1}, x_{0}\right)+2\left(1-2^{-k}\right)\left\{C_{3} E\left(R_{1}, x_{0}\right)+C_{3}\left(2 C_{2} \tau^{-\alpha}+1\right) \gamma_{0} R_{1}^{\alpha}\right\} .
\end{aligned}
$$

We prove the claim by using induction over $k \in \mathbb{N} \cup\{0\}$.

Firstly, note that for $k=0$ both (7.17) and (7.18) are trivially fulfilled. Assume (7.17) and (7.18) hold for $k \in \mathbb{N} \cup\{0\}$. Observing (7.15) and (7.16), the assumption (7.18) implies

$$
M\left(\tau^{k} R_{1}, x_{0}\right) \leq M_{0} .
$$

By the choice of $M_{0}(7.19)$ yields (7.11) for $R=\tau^{k} R_{1}$. Hence from (7.12) with $R=\tau^{k} R_{1}$ we infer

$$
E\left(\tau^{k+1} R_{1}, x_{0}\right) \leq \tau^{\beta} E\left(\tau^{k} R_{1}, x_{0}\right)+C_{2} \gamma_{0} \tau^{\alpha k} R_{1}^{\alpha}
$$

Now, estimating the first term by the assumption (7.17) taking into account (7.6), we arrive at

$$
\begin{aligned}
E\left(\tau^{k+1} R_{1}, x_{0}\right) & \leq \tau^{\beta(k+1)} E\left(R_{1}, x_{0}\right)+2 \tau^{\beta-\alpha}\left(1-2^{-k}\right) \tau^{\alpha k} C_{2} \gamma_{0} R_{1}^{\alpha}+\tau^{\alpha k} C_{2} \gamma_{0} R_{1}^{\alpha} \\
& \leq \tau^{\beta(k+1)} E\left(R_{1}, x_{0}\right)+\left(2-2^{-k}\right) \tau^{\alpha k} C_{2} \gamma_{0} R_{1}^{\alpha}
\end{aligned}
$$

which results in (7.17) for $k+1$.

It remains to verify (7.18) for $k+1$. In fact, by means of (7.13) with $R=\tau^{k} R_{1}$ together with the assumption (7.17) and (7.18) we estimate

$$
\begin{aligned}
& M\left(\tau^{k+1} R_{1}, x_{0}\right) \\
& \leq M\left(\tau^{k} R_{1}, x_{0}\right)+C_{3}\left(E\left(\tau^{k} R_{1}, x_{0}\right)+\gamma_{0} \tau^{\alpha k} R_{1}^{\alpha}\right) \\
& \leq M\left(R_{1}, x_{0}\right)+2\left(1-2^{-k}\right)\left\{C_{3} E\left(R_{1}, x_{0}\right)+C_{3}\left(2 C_{2} \tau^{-\alpha}+1\right) \gamma_{0} R_{1}^{\alpha}\right\} \\
& \quad+C_{3}\left(\tau^{\beta k} E\left(R_{1}, x_{0}\right)+2 \tau^{\alpha(k-1)} C_{2} \gamma_{0} R_{1}^{\alpha}+\gamma_{0} \tau^{\alpha k} R_{1}^{\alpha}\right) \\
& \leq M\left(R_{1}, x_{0}\right)+2\left(1-2^{-k}\right)\left\{C_{3} E\left(R_{1}, x_{0}\right)+C_{3}\left(2 C_{2} \tau^{-\alpha}+1\right) \gamma_{0} R_{1}^{\alpha}\right\} \\
& \quad+2^{-k}\left(C_{3} E\left(R_{1}, x_{0}\right)+C_{3}\left(2 C_{2} \tau^{-\alpha}+1\right) \gamma_{0} R_{1}^{\alpha}\right) \\
& =M\left(R_{1}, x_{0}\right)+2\left(1-2^{-k-1}\right)\left\{C_{3} E\left(R_{1}, x_{0}\right)+C_{3}\left(2 C_{2} \tau^{-\alpha}+1\right) \gamma_{0} R_{1}^{\alpha}\right\} .
\end{aligned}
$$

Whence, (7.18) for $k+1$.

This implies the following 
Theorem 7.3. Let $\boldsymbol{F} \in \mathcal{M}^{2, \lambda}, 1<\lambda<3$. Let $\boldsymbol{B} \in \dot{W}^{1,2}$ be a suitable weak solution to (3.1). Then there exists $\rho_{0}>0$ such that $\Sigma(\boldsymbol{B}) \subset B_{\rho_{0}}$.

As a consequence of Theorem 7.3 we get

Corollary 7.4. Let $\boldsymbol{f} \in L^{6 / 5} \cap L^{2}$ and $\boldsymbol{g} \in L^{2} \cap L^{q}$ for some $3<q<+\infty$. Let $(\boldsymbol{u}, p, \boldsymbol{B})$ be a suitable weak solution to the steady Hall-MHD system. Then there exists $\rho_{0}>0$ such that $\boldsymbol{B}$ is Hölder contiuous in $\left\{x:|x|>\rho_{0}\right\}$. In particular, $\Sigma(\boldsymbol{B})$ is a compact set of Hausdorff dimension at most one.

Proof To prove the corollary we only need to verify that $-\boldsymbol{B} \times \boldsymbol{u}+\boldsymbol{g} \in \mathcal{M}^{2, \lambda}$ for some $1<\lambda<3$. Then the assertion follows immediately from Theorem 7.3 with $\boldsymbol{F}=-\boldsymbol{B} \times \boldsymbol{u}+\boldsymbol{g} \in \mathcal{M}^{2, \lambda}$.

First, using Hölder's inequality, we find $\|\boldsymbol{g}\|_{2, B_{R}}^{2} \leq c R^{3(q-2) / q}\|\boldsymbol{g}\|_{q}$ for every ball $B_{R} \subset \mathbb{R}^{3}$, which implies $\boldsymbol{g} \in \mathcal{M}^{2,3(q-2) / q}$. Owing to $3<q<+\infty$ we have $1<\frac{3(q-2)}{q}<3$. Next, as $\boldsymbol{V}=\boldsymbol{B}+\boldsymbol{\omega} \in L^{6}+L^{2}$ and $\boldsymbol{u} \in L^{6}$, we see that $-\boldsymbol{V} \times \boldsymbol{u}+(\boldsymbol{f}+\boldsymbol{g}) \in L^{3 / 2}+L^{3}+L^{2}$. By Calderón-Zygmund's inequality it follows that $\nabla \boldsymbol{V} \in W^{1,3 / 2}+W^{1,3}+W^{1,2}$. By means of Sobolev's embedding theorem we get

$$
\begin{aligned}
\|\boldsymbol{\omega}\|_{3, B_{2}\left(x_{0}\right)} & \leq\|\boldsymbol{V}\|_{3, B_{2}\left(x_{0}\right)}+\|\boldsymbol{B}\|_{3, B_{2}\left(x_{0}\right)} \\
& \leq c\left(\|\boldsymbol{\omega}\|_{2}\|\boldsymbol{u}\|_{6}+\|\boldsymbol{B}\|_{6}\|\boldsymbol{u}\|_{6}+\|\boldsymbol{f}+\boldsymbol{g}\|_{2}\right)
\end{aligned}
$$

for all $x_{0} \in \mathbb{R}^{3}$, with an absolute constant $c>0$. As $\nabla \cdot \boldsymbol{u}=0$, we obtain

$$
\|\boldsymbol{u}\|_{10, B_{1}\left(x_{0}\right)} \leq c\left(\|\boldsymbol{u}\|_{6}\left(1+\|\boldsymbol{\omega}\|_{2}+\|\boldsymbol{B}\|_{6}\right)+c\|\boldsymbol{f}+\boldsymbol{g}\|_{2} \quad \forall x_{0} \in \mathbb{R}^{3} .\right.
$$

Accordingly, $\boldsymbol{B} \times \boldsymbol{u} \in \mathcal{M}^{2,7 / 5}$. This completes the proof of the corollary.

Acknowledgements Chae was partially supported by NRF grants 2006-0093854 and 2009-0083521, while Wolf has been supported by the Brain Pool Project of the Korea Federation of Science and Technology Societies (141S-1-3-0022).

\section{References}

[1] M. Acheritogaray, P. Degond, A. Frouvelle and J-G. Liu, Kinetic formulation and global existence for the Hall-Magnetohydrodynamic system, Kinetic and Related Models, 4 (2011), pp. 901-918.

[2] L. Caffarelli, R. Kohn and L. Nirenberg, Partial Regularity of suitable weak solutions of the Navier-Stokes equations, Comm. Pure Appl. Math. 35 (1982), pp. 771-831.

[3] S. Campanato, Equazioni ellittiche del secondo ordine e spazi $\mathcal{L}^{2, \lambda}$, Ann. Mat. Pura e Appl. 69 (1965), pp. 321-380.

[4] D. Chae, P. Degond and J-G. Liu, Well-posedness for Hall-magnetohydrodynamics, Ann. Inst. Henri Poincare-Analyse Nonlineaire 31 (2014), pp. 555-565. 
[5] D. Chae and J. Lee, On the blow-up criterion and small data global existence for the Hall-magnetohydrodynamics. J. Differential Equations 256 (2014), no. 11, pp. 3835-3858.

[6] D. Chae and M. Schonbek, On the temporal decay for the Hallmagnetohydrodynamic equations. J. Differential Equations 255 (2013), no. 11, pp. 3971-3982.

[7] D. Chae and S. Weng, Singularity formation for the incompressible Hall-MHD equations without resistivity, Ann. Inst. Henri Poincare-Analyse Nonlineaire, (in print) http://dx.doi.org/10.1016/j.anihpc.2015.03.002.

[8] J. Dreher, V. Runban and R. Grauer, Axisymmetric flows in Hall-MHD: a tendency towards finite-time singularity formation. Physica Scripta 72 (2005), pp. 451-455.

[9] J. Fan, S. Huang and G. Nakamura, Well-posedness for the axisymmetric incompressible viscous Hall-magnetohydrodynamic equations. Appl. Math. Lett. 26 (2013), no. 9, pp. 963-967.

[10] T.G. Forbes, Magnetic reconnection in solar flares, Geophys. Astrophys. Fluid Dyn. 62 (1991), pp.15-36.

[11] M. Giaquinta, Multiple integrals in the calculus of variations and nonlinear elliptic systems, Ann. of Math. studies 105, Princeton Univ. press, Princeton, New Jersey, 1983.

[12] H. Homann and R. Grauer, Bifurcation analysis of magnetic reconnection in HallMHD systems. Physica D 208 (2005), pp. 59-72.

[13] A. Kufner and A. Wannebo, An interpolation inequality involving Hölder norms, Georgian Math. J. 2, no. 6 (1995), pp. 603-612.

[14] O. A. Ladyzehnskaya and G. A. Seregin, On partial regularity of sutiable weak solutions to the three-dimensional Navier-stokes equations, J. Math. Fluid Mech. 1, no. 4 (1999), pp. 356-387.

[15] M. J. Lighthill, Studies on magneto-hydrodynamic waves and other anisotropic wave motions, Philos. Trans. R. Soc. Lond. Ser. A 252 (1960), pp. 397-430.

[16] F. H. Lin, A new proof of the Caffarelli-Kohn-Nirenberg theorem, Comm. Pure Appl. Math. 51 (1998), pp. 241-257.

[17] J. M. Polygiannakis and X. Moussas, A review of magneto-vorticity induction in Hall-MHD plasmas, Plasma Phys. Control \& Fusion 43 (2001), pp.195-221.

[18] V. Scheffer, Partial regularity of solutions to the Navier-Stokes equations, Pacific J. Math. 66 (1976), pp. 535-552. 
[19] D.A. Shalybkov, V.A. Urpin, The Hall effect and the decay of magnetic fields, Astron. Astrophys. (1997), pp. 685-690.

[20] H. Miura and D. Hori, Hall effects on local structure in decaying MHD turbulence, J. Plasma Fusion Res. 8 (2009), pp. 73-76.

[21] A. N. Simakov and L. Chacón, Quantitative, analytical model for magnetic reconnection in Hall magnetohydrodynamics, Phys. Rev. Lett. 101 (2008), 105003.

[22] M. Wardle, Star formation and the Hall effect, Astrophys. Space Sci. 292, (2004) pp. 317-323.

[23] J. Wolf, On the local regularity of suitable weak solution to the generalized NavierStokes equations, Ann. Univ. Ferrara, DOI 10.1007/s11565-014-0203-6. 